\title{
The PIN-Diode Beam Loss Monitor System At HERA
}

\author{
Kay Wittenburg \\ Deutsches Elektronen Synchrotron, DESY, Hamburg
}

\begin{abstract}
The PIN-Diode Beam Loss Monitors (BLMs) developed at DESY were the subject of the Faraday Cup Award 2000. This talk presents a general overview of the system at HERA, its use and the experience with these BLMs at DESY.
\end{abstract}

\section{INTRODUCTION}

The PIN-Diode BLMs were originally designed to meet the special conditions in HERA, which consists of a superconducting $920 \mathrm{GeV} / \mathrm{c}$ proton ring and a $30 \mathrm{GeV} / \mathrm{c}$ electron (positron) ring in the same $6.3 \mathrm{~km}$ long tunnel. The BLMs for the HERAproton-ring (HERAp) must fulfil the following requirements:

- They have to measure losses with sufficient sensitivity and response to prevent beam loss induced quenching of the superconducting magnets.

- The dynamic range of the monitors must exceed several decades in order to measure losses during beam lifetimes of hundreds of hours as well as the much greater losses that may quench superconducting magnets.

- They must be insensitive to the synchrotron radiation of the adjacent electronring (HERAe). The dose rate at the monitor position was expected to be about $10^{6} \mathrm{rad} /$ year. For usual loss monitors such as ion chambers, this dose is strong enough to create signals close to those expected from a dangerous beam loss.

- Their radiation hardness must allow a monitor-lifetime of a few years of HERA operation.

The idea of using PIN photodiodes for beam loss detection was first discussed in 1987 (Ref. 1). In 1989 the coincident readout of two diodes was incorporated to suppress the synchrotron radiation background (Ref. 2, 3). First tests were made in the following years, which led to the final readout electronics of these detectors. The BLM system (250 units) was installed in 1993 in HERAp and is still running very reliably, as a very important part of the quench protection system for the superconducting magnets and as a useful diagnostic tool. A second BLM system was installed in 1994 in HERAe to study a serious beam current limitation, characterized by very short lifetimes of the electron beam. A total of 214 PIN Diode BLMs were installed around the entire ring to locate the regions and diagnose the causes of the beam losses. Their successful results led to a satisfactory increase of the stored beam current and of the electron beam lifetime. 
During the 7 years of operation, the failure rate of the BLMs has been less than $1 \%$ / year and the whole readout system requires a minimum of intervention.

\section{THE BLM SYSTEM}

\section{The Detector}

A shower of charged and neutral particles is created if a lost particle from a highenergy beam hit the beam pipe of the accelerator. At very high energies, as in HERA, the shower penetrates the beam pipe and the surrounding magnet and particles leave the surface of the material. A small number of these particles will reach the BLM positioned on top of the magnet and can be detected. The monitors consist of two reverse biased PIN-photodiodes, mounted face-to-face. Charged particles which cross both diodes produce coincident signals with a high efficiency while the efficiency of coincident signals from photons from the synchrotron radiation is very small. The dimensions of the photodiodes are $2.75 \times 2.75 \mathrm{~mm}^{2}$ (Siemens BPW 34) or $7.5 \times 20$ $\mathrm{mm}^{2}$ (Hamamatsu S2662) depending on the sensitivity required at each position.

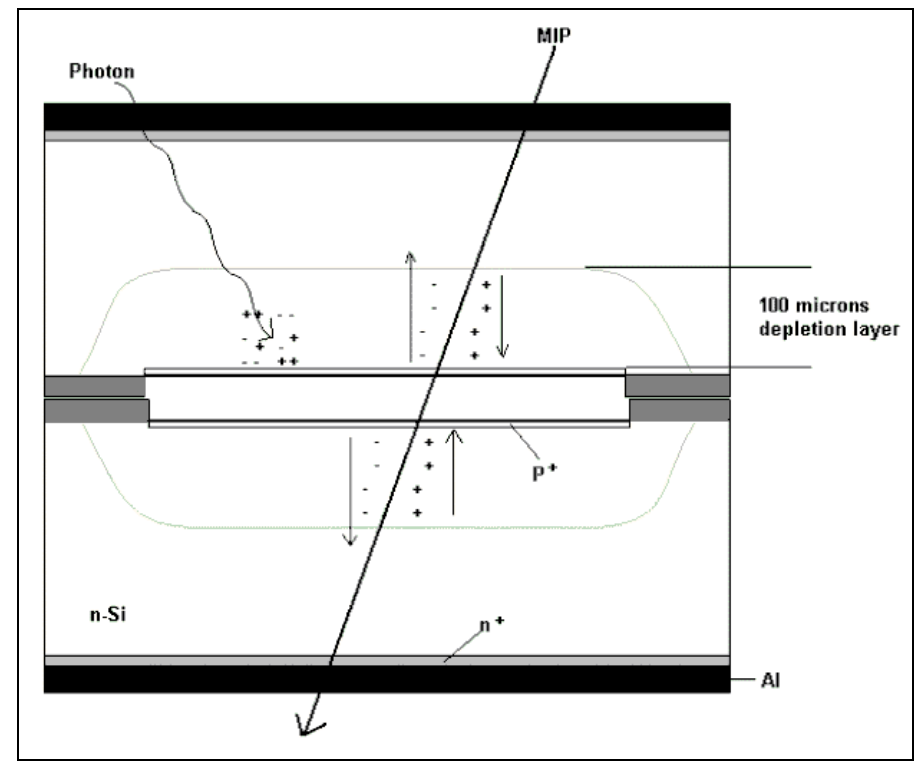

FIGURE 1. The PIN diodes mounted face to face

The depletion layer of about $100 \mu \mathrm{m}$ is a result of a reversed bias voltage of $24 \mathrm{~V}$. The required energy for a creation of an electron - hole pair is about $3.6 \mathrm{eV}$ in Silicon. The energy deposed by a minimum ionizing particle (MIP) in Silicon is about 3.6 $\mathrm{MeV} / \mathrm{cm}$. Therefore about 10000 electron - hole pairs are created by a MIP in the depletion layer, which are separated by the bias voltage and then detected by a sensitive amplifier. 


\section{The amplifier}

The main idea of the beam loss measurement in HERA was to count beam losses with a maximum frequency of the bunch spacing $(10.4 \mathrm{MHz})$. Therefore the main purpose of the amplifier was to detect signal $\geq 10000 \mathrm{e}^{-}$and deliver short TTL signals $(\approx 50 \mathrm{~ns})$ at the output. The linearity of the amplifier is of no interest.

One of the important requirements of the amplifier was the insensitivity to radiation damage. Therefore the first amplification stages (preamplifier) were built with fast bipolar transistors followed by a simple video amplifier chip. This circuit was successfully tested up to $2 \cdot 10^{8} \mathrm{rad}$ (Refs. 4-6). The threshold of the following comparator can be adjusted individually for each channel by potentiometers. This reduces the dark count rate of each channel. The potentiometers were adjusted for a dark count rate of about $1 \mathrm{kHz}$ for each channel. A TTL signal is created at the TTL driver when both comparators are above the threshold in coincidence. The coincident dark count rate is than less than $0.01 \mathrm{~Hz}$. Therefore the dynamic range of this amplifier is greater than $10^{9}$.

Each channel has a $5 \mathrm{~V}$ test input, which enables its comparator. Therefore the count rate of the other channel is applied on the TTL driver. This feature allows a simple and complete system test of the whole readout chain of each monitor. The amplifier can drive about $100 \mathrm{~m}$ of good coaxial cable.

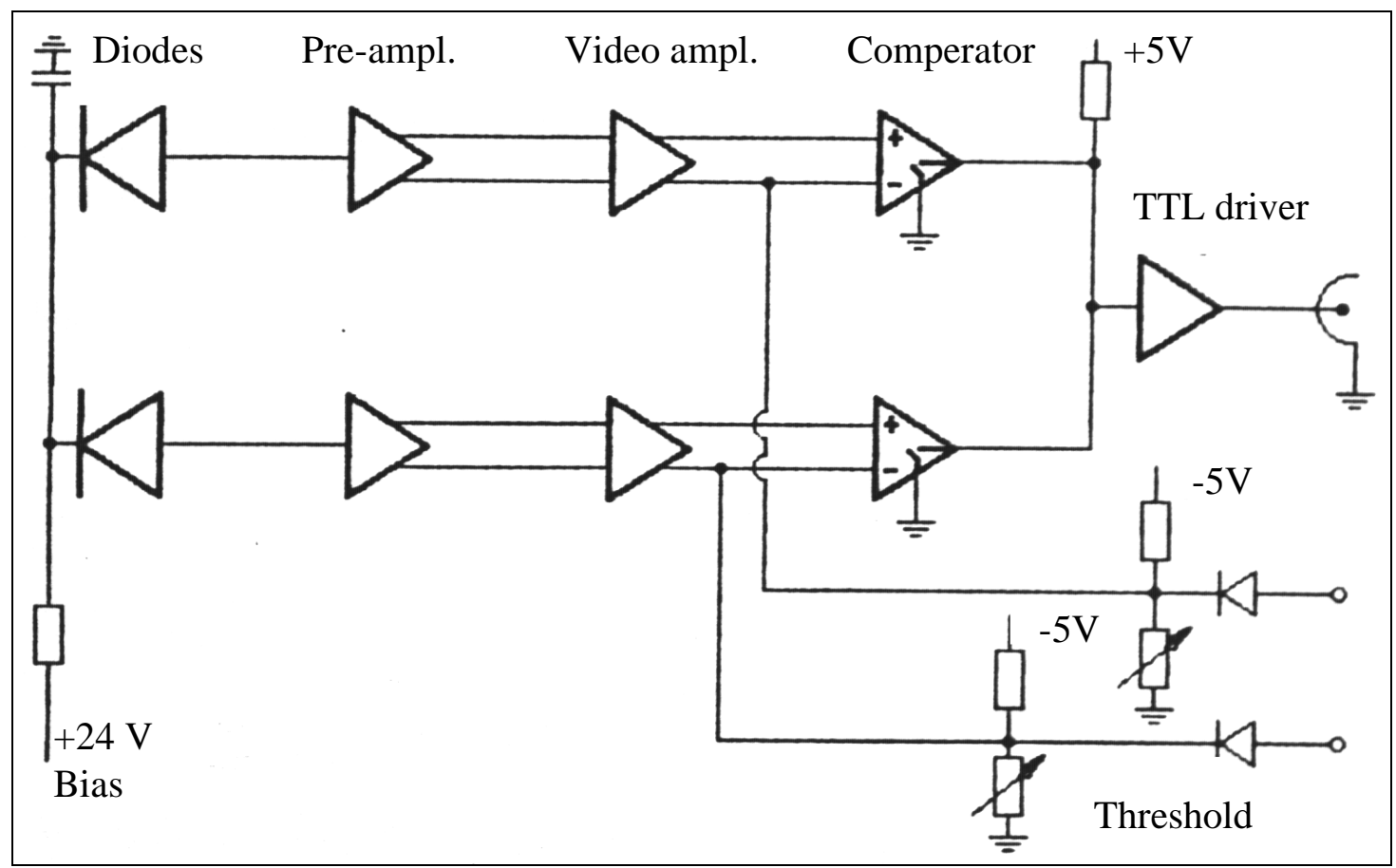

FIGURE 2. The PIN diode amplifier 


\section{The counting module}

The input of each channel of the counting module is a fast counter, followed by two different data ring-buffers, a short-term and a long-term memory. The short term memory integrates the counts over an interval of $5.2 \mathrm{~ms}$. This time was chosen to be shorter than the expected cryogenic time constant of the superconducting magnets. The last 128 time intervals are always stored in the short-term memory. Every 128 * $5.2 \mathrm{~ms}$ the mean value of the short-term memory is transferred to the long-term memory, which stores the last 128 mean values.

If the number of counts within $5.2 \mathrm{~ms}$ reach a software-defined threshold, an alarm signal is applied to the HERA quench protection system (Ref 7). In the case that more than 5 alarms are created by the BLMs, the beam is dumped within one turn and a freeze signal stops the data taking after about $30 \mathrm{~ms}$. The data in the memories are readout and stored in an archive file. This allows a post mortem analysis of the beam losses within the last $666 \mathrm{~ms}$ (short-term memory) and, on a more coarse-meshed scale, within the last $85 \mathrm{~s}$ (long-term memory).

The interface to the HERA control system is provided by the DESY-standard SEDAC fieldbus. The current loss rates of all BLMs as well as archives of all the alarm cases are visible in the HERA control room.

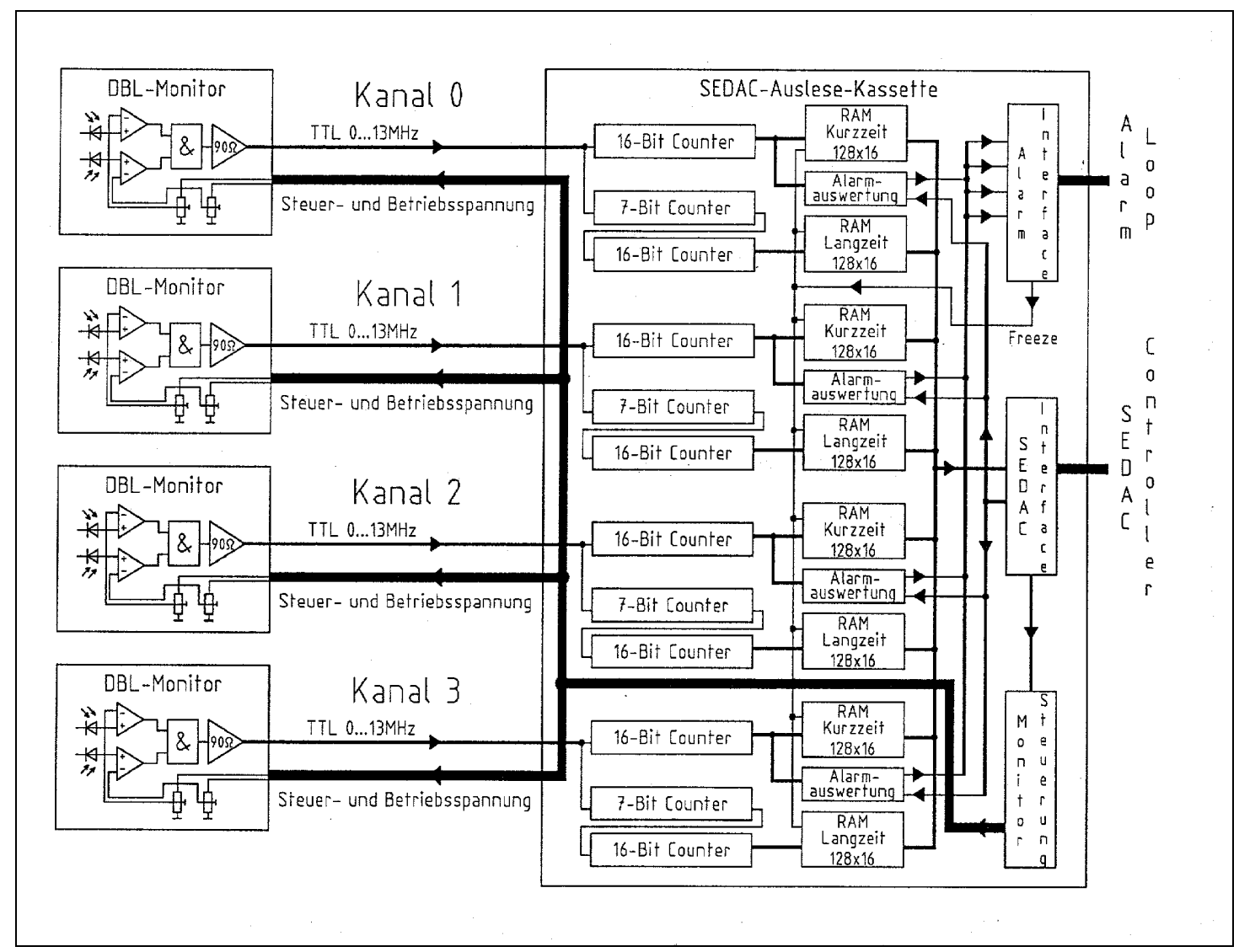

FIGURE 3. The SEDAC BLM-counter module. Up to four BLMs can be connected to one module. 


\section{The Efficiency of the BLM}

1) Efficiency for MIPs:

The efficiency of the BLM for MIPs was measured by Refs 8, 9. It was found to be about $30-35 \%$ for the coincident readout of the BLM. The uncertainty might be a result of non-precise alignment of the two sensitive areas of the two diodes.

2) Efficiency for beam losses:

The most likely positions for beam losses in a proton accelerator are the quadrupole magnets, where the beam size is maximal, and the aperture limits, which are mainly defined by collimators. The efficiencies of the BLM at these locations were determined by Monte Carlo simulations (Geisha 8, Ref. 10). The distribution of the energy deposition and of the MIPs leaving the cryostat was calculated, assuming a point like proton loss distribution in the middle of the superconducting quadrupole. Fig. 4 shows that the shower outside of the cryostat is distributed over some meters and that it is radially symmetric. This is valid for proton energies between 40 and 820 $\mathrm{GeV} / \mathrm{c}$. The position of the BLM was chosen such that it detects parts of the long tail of the shower. This provides an efficiency that is almost independent of the exact location of the beam loss within the first half ${ }^{1}$ of the quadrupole.

Fig. 5 a shows the calibration of the BLM with a small $\left(7.3 \mathrm{~mm}^{2}\right)$ diode located on the superconducting quadrupole in HERA. The efficiency depends linearly on the energy of the lost protons. One count corresponds to about $1.8 \cdot 10^{4}$ lost protons at 820 $\mathrm{GeV} / \mathrm{c}$. An even better efficiency can be achieved at the collimators (Fig. 5b). The efficiency is about 60 lost protons/count achieved with the small diode. A gain in efficiency can easily be achieved by using larger diodes. One can expect to detect nearly every lost proton in the collimator with the $7.5 \times 20 \mathrm{~mm}^{2}$ diode.

This kind of calibration was performed for all locations of BLMs in HERAp (Ref. 11). Assuming that all possible positions of proton loss are occupied by BLMs, the lifetime of the beam can be measured by adding all loss rates and by knowing the beam current. The resulting lifetime is found to be always a factor 2 lower than that calculated from the decrease of the beam current. This is valid for short lifetimes of a few hours as well as for long lifetimes of more than 1000 hours. Therefore the calibration might be a factor two too large if the assumption from above is right. It was taken into account for the threshold setting for shielding the superconducting magnets from beam loss induced quenches.

\section{EXPERIENCES WITH THE PIN DIODE BLMS IN HERA P}

HERA started operation at the beginning of 1993 with the BLM system not connected to the quench protection and alarm system. One third of the BLMs were connected in week 26 and the remaining $2 / 3$ were connected in week 37. Fig. 6a shows that the number of beam loss induced quenches (gray lines) was drastically reduced by the help of this BLM system (see also Ref. 12). Some additional beam loss induced beam dumps (Fig. 6, black lines), quenches and unexpected events (Fig. 6, dashed and

\footnotetext{
${ }^{1}$ Note that the beam is focused after the middle of the quadrupole so that no losses should occur behind it. In the defocusing case,
} the beam has its smallest envelope so that there shall be no losses at all. 
hatched lines) occurred with the increasing beam current of HERA during the following years are discussed in the following.

Errors (dashed lines in Fig. 6): The BLM system has worked very reliably over the past 7 years of operation. There were a handful of unnecessary beam dumps due to a malfunction of the SEDAC system in 1994 and 1996. Another cluster of 8 unintentional beam dumps occurred in the middle of 1996 due to a malfunction of the alarm system, by which the threshold of allowed alarms was set arbitrarily between 0 and 255.

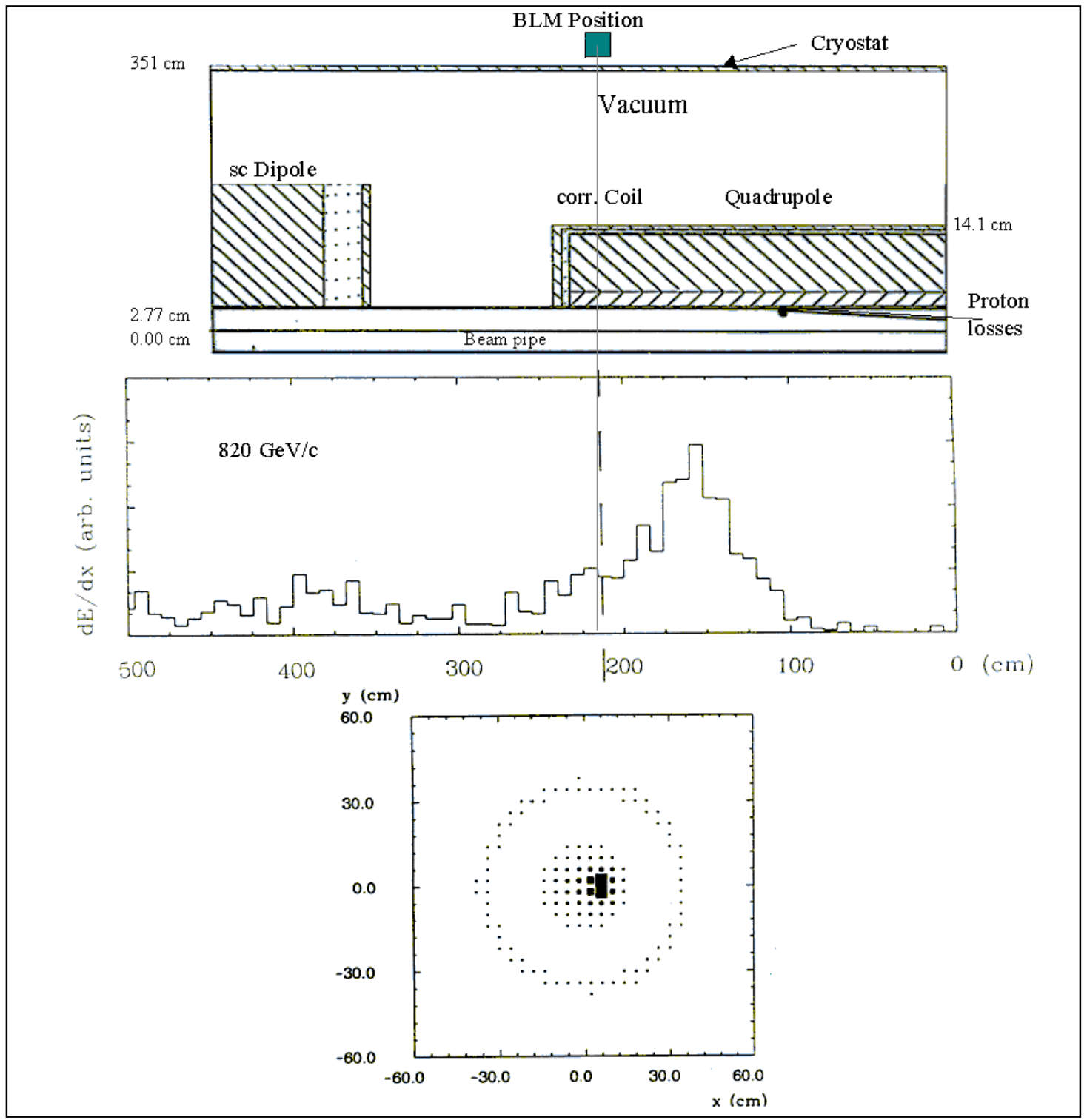

FIGURE 4. Longitudinal and radial energy/MIP distribution in the surface of the cryostat after proton losses in the middle of the superconducting quadrupole 


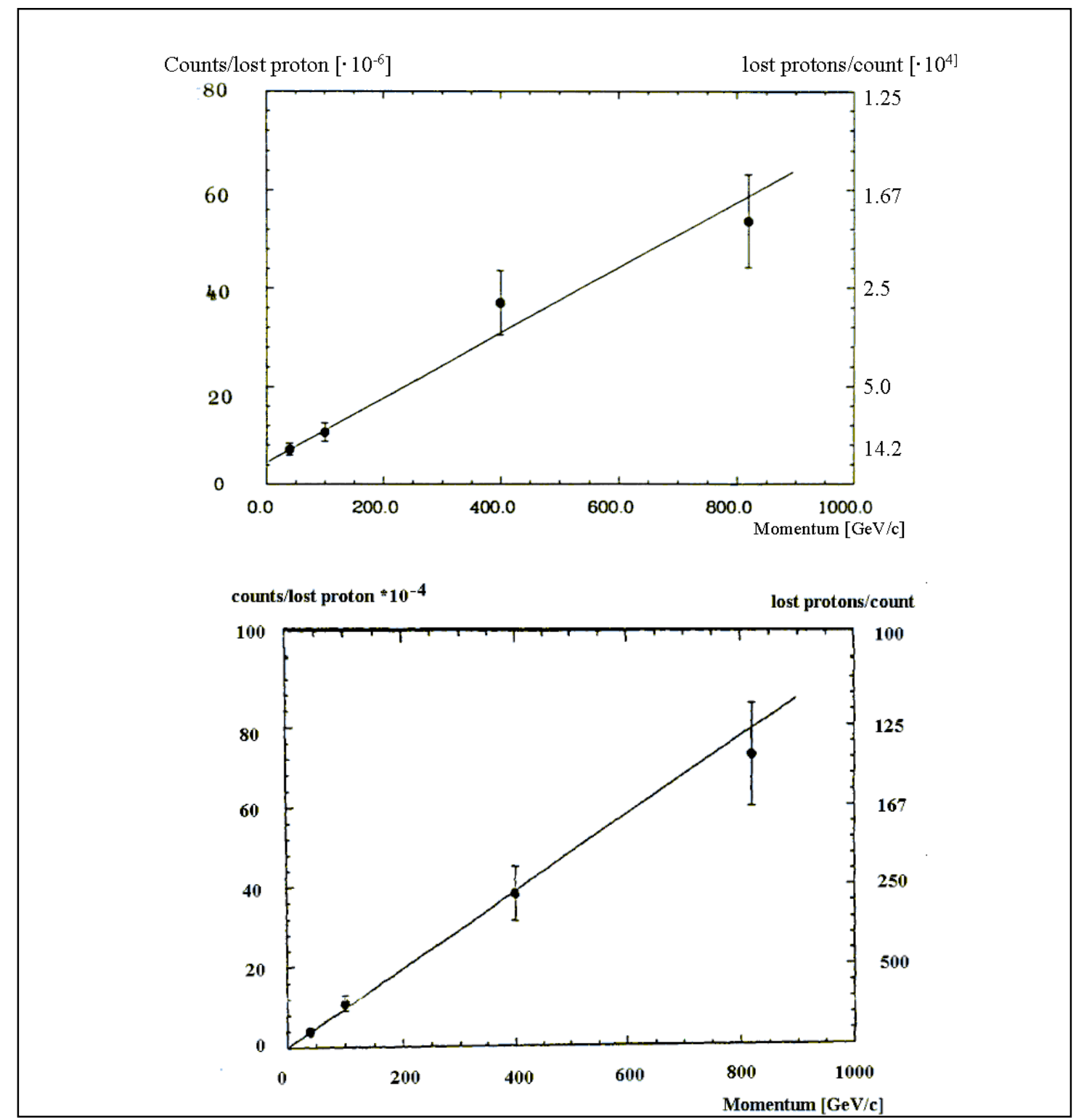

FIGURE 5. a) Calibration of the BLM at the superconducting quadrupole magnets. b) Calibration at the proton collimator. Both cases were calculated for small diodes. The efficiency to MIPs is already included (see above).

However, in both cases the BLM system was not the cause of these events. Due to the simplicity of the counting system there have been no malfunctions of the system during the years of operation. The internal systems check (see above) gives the possibility of an automatic system check using software, which is not yet implemented. But the results of the yearly checks (by 'hand') gave no impetus for such a routine, because only a minimum of investigation was required.

Beam loss induced beam dumps (black lines in Fig. 6): The most frequent events were due to the 'normal' operation of the BLM system. Fig. 7, 8 show typical views of the behavior of the system. Both figures display archived data after a beam loss induced alarm. The upper picture of each figure shows the losses around the entire HERA 
proton ring while the middle shows the short term memory of 4 selected BLMs and the lower picture shows the long-term memory of the same BLMs. Fig. 7 shows very long-term losses due to orbit steering by the operators who were trying to reduce some larger losses in the warm North region of HERA. Unfortunately the loss rate increased at other locations. Then the BLM on the superconducting quadrupole WL162 was the $5^{\text {th }}$ BLM to exceed the threshold (horizontal dashed line) and the beam was immediately dumped. The BLMs in the right corner of the upper picture are monitors between the beam dump kickers and the beam dump. They receive a huge burst of lost particles after the beam dump kickers fire and then detect the decay of radioactivity induced by the lost particles. Fig. 8 shows a much faster increase of the beam loss due to a trip of the $208 \mathrm{MHz}$ RF-system. A large number of BLMs reached their thresholds within $30 \mathrm{~ms}$ initiating a beam dump. The long-term memory shows that there were no losses during the normal operation of HERA. Typically in normal operation the loss rates in the superconducting area are less than 1 count / $5.2 \mathrm{~ms}$ while in the warm regions (aperture limits) the rate is about 10 counts / $5.2 \mathrm{~ms}$. Most of the beam loss induced beam dumps had a time scale between these two examples. But there were even faster events with a time scale of less than $5 \mathrm{~ms}$.

$5 \mathrm{~ms}$ events (hatched lines in Fig. 6): The signature of such an event is that a lot of BLMs show only one line exceeding the thresholds before the beam dump. A few of these very fast beam losses quenched one or more superconducting magnets. The reason for these events was not fully understood. However, since in 1998 more and faster status information of magnets and RF-systems were added to the quench protection system, the frequency of beam loss induced beam dumps and $5 \mathrm{~ms}$ events was drastically reduced. The remaining $5 \mathrm{~ms}$ events were probably due to huge electron beam losses, which crosstalked to the proton beam loss monitors.

Beam loss induced quenches (gray lines in Fig. 6): There are three different types of beam loss induced quenches, which are missed by the BLM system. 1) The $5 \mathrm{~ms}$ events that were too fast for the integration time of the system (see above), 2) the losses occurred in less than 5 magnets. In these very rare events, losses occur in two or three superconducting magnets without losses in the aperture limits in the warm regions of HERA. These events occurred only during the energy ramp of HERA, where the collimators had a large aperture. Tighter settings can avoid these quenches, 3) Injection failures. An injection train of maximum $60 \times 10^{11}$ protons in HERA can be missteered due to technical or operator errors. Sometimes all protons are lost in a small area within one turn. If this happens in the superconducting part of HERA, these magnets quench. However, no BLM system can prevent this.

The proton loss rates observed by the BLM, which led to quenches of the corresponding magnets, were always about a factor $2-3$ above the threshold of the BLM. But note that the threshold was set to about a factor 10 below the expected critical loss rate for the superconducting quadrupoles. 


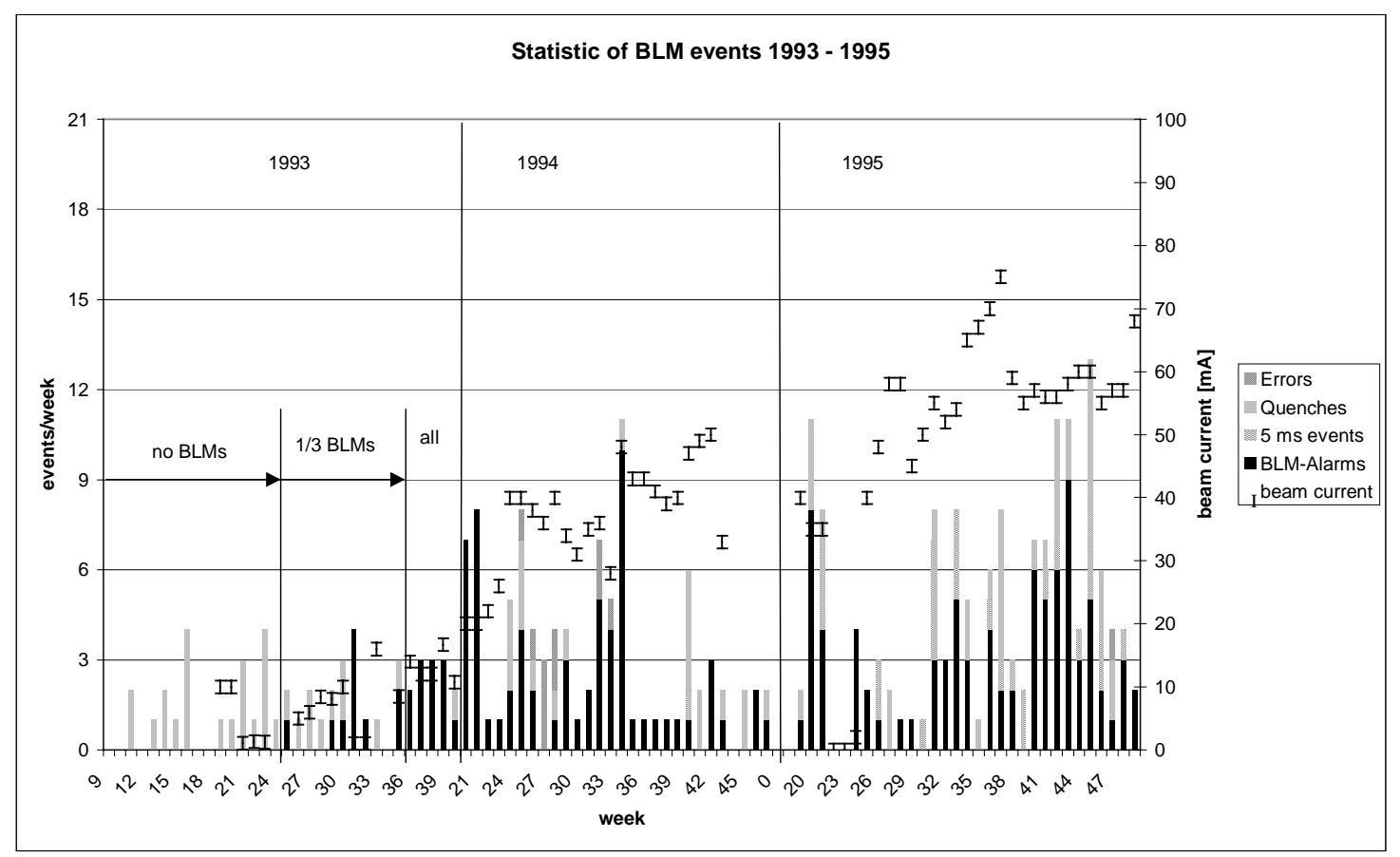

a)

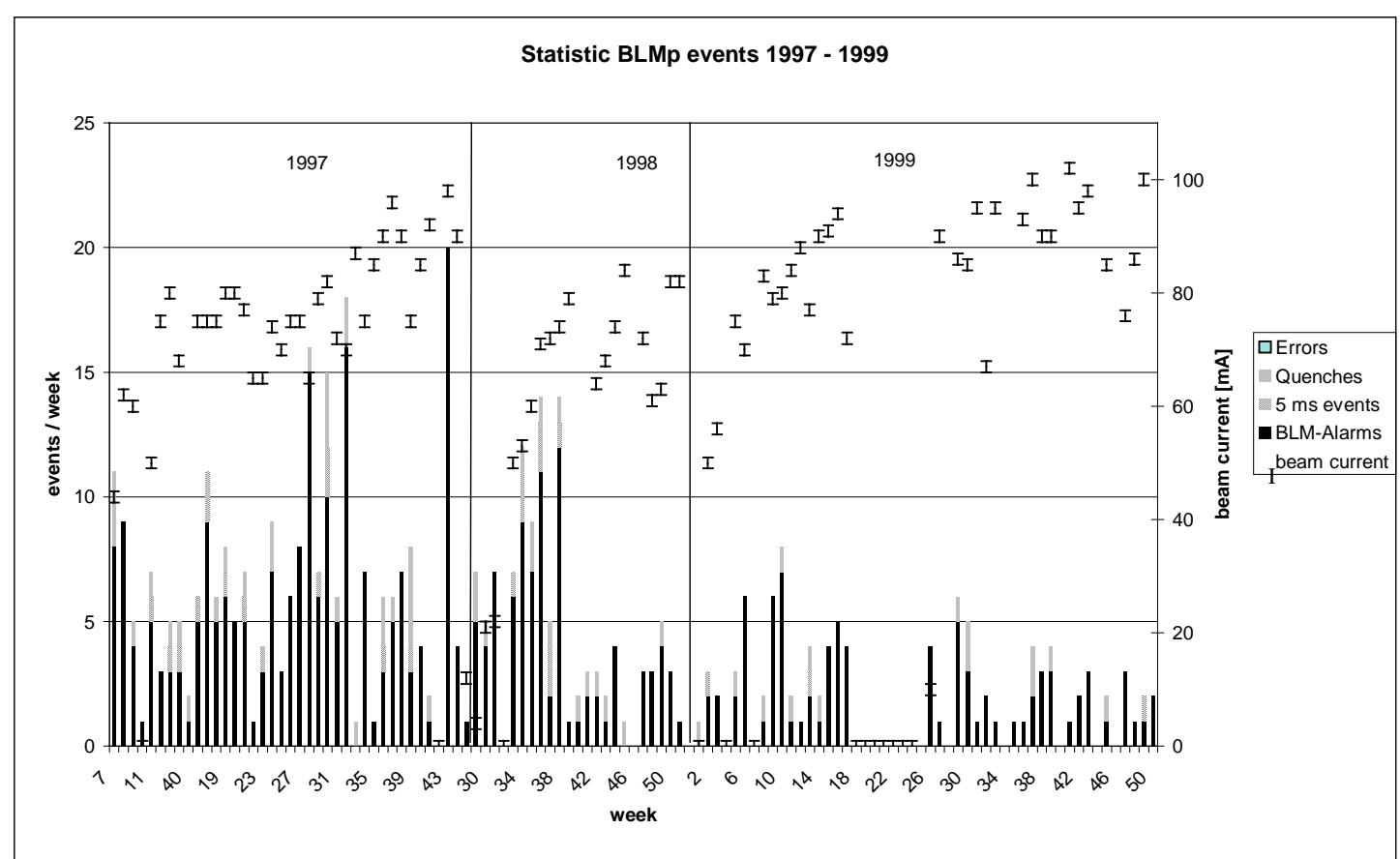

b)

FIGURE 6. Statistic of beam loss induced beam dumps, quenches, 5 ms events and errors of the BLM system in 1993 -95 (a) and 1997-99 (b). 

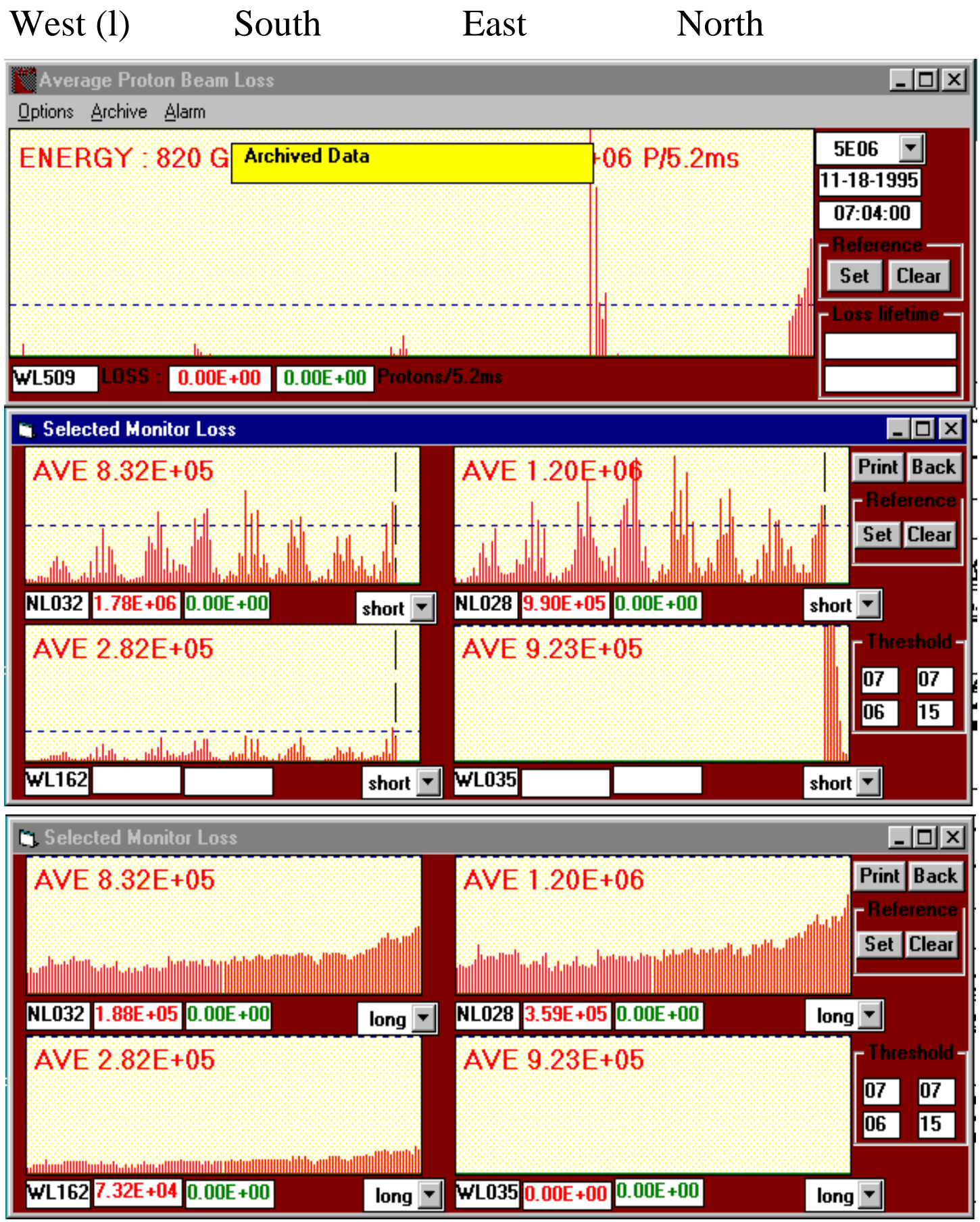

FIGURE 7. Proton losses during orbit steering. The losses increased slowly during more than $85 \mathrm{~s}$. The protons circulate from West (left) to South, East, North and West (right). 


\section{West (1) South East North}

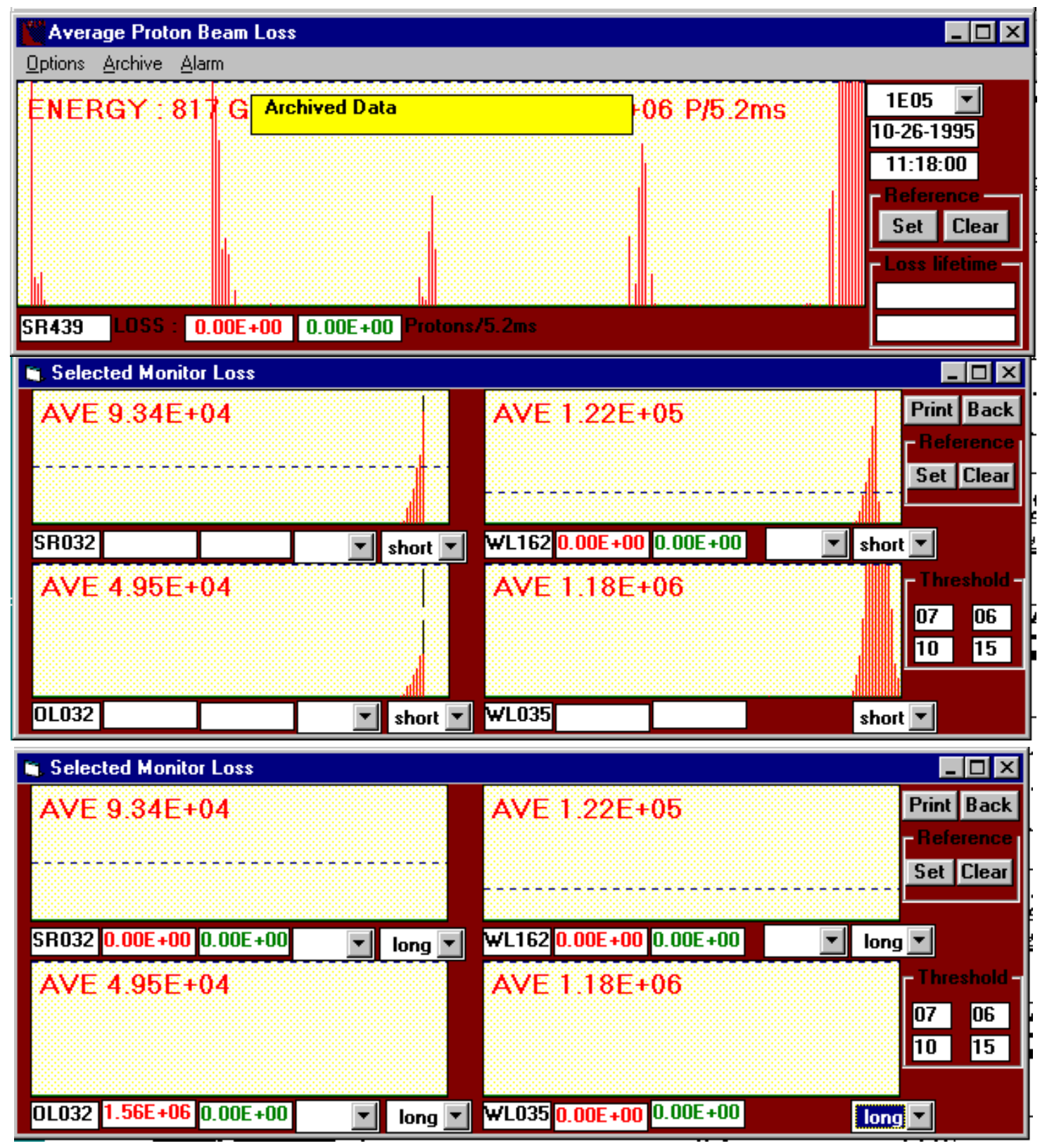

FIGURE 8. Proton losses during a trip of the $208 \mathrm{MHz}$ RF - System. The losses increased fast within about $30 \mathrm{~ms}$. The protons circulate from West (left) to South, East, North and West (right). 


\section{EXPERIENCES WITH THE PIN DIODE BLMS IN HERA E}

The aim of the HERAe BLM system is to observe the position of beam losses around the ring. The readout electronic is slightly different to that of the proton ring. The integration time can be set between 0.1 and 100s and there is no connection to the alarm loop. The BLMs are mounted on the inside of the vacuum chamber of the electron ring just behind each focusing quadrupole. This specific position is required for the localization of targets in the vacuum (e.g. residual gas, micro particles, dust) within only one, the previous, FODO cell. This is very useful to study lifetime phenomena in HERAe, especially the trapping of micro particles (Ref. 13-14) ${ }^{2}$.

The efficiencies of the BLM at their positions were determined by particle tracking and by shower simulations. In the case of the electron beam, the losses are determined typically by Bremsstrahlung on residual gas molecules in the vacuum. The path of an electron after a Bremsstrahlung interaction depends on the amount of energy lost and on the position of the target in the ring. This very good position resolution for the target in the machine is demonstrated in Fig. 9a-d, where a short-term decrease and a long-term decrease of the lifetime are shown. In each case, only one BLM in the arcs shows an increased loss rate. The loss rate of this BLM followed very precisely the inverse lifetime of this event. With the help of the BLM system one could observe even travelling targets along parts of the ring. The loss mechanism, the detection and the monitors are discussed in more detail in Refs. 15 - 17.

The coincident readout of the two PIN photodiodes helps to reduce the background of the synchrotron radiation, thus allowing the BLMs to be useful for electron accelerators. However, a synchrotron radiation photon can create a Photo- or Compton- electron in one diode, which may reach the other diode, creating a coincident signal. A thin layer of metal between the diodes can avoid this, since these electrons are absorbed within this layer. The signal to background ratio at HERAe was improved by a factor 20 with the help of such a layer and some additional shielding (Ref. 18, 19).

\section{OTHER APPLICATIONS}

1) Vacuum: The BLMs in HERAe are sensitive to the energy loss of the electrons due to Bremsstrahlung. Therefore their count rates follow the vacuum pressure distribution in the ring (Ref .16). The BLMs can detect especially vacuum leaks easily and quickly.

Studies by the authors of Ref. 20 for LHC have shown that the sensitivity of the BLMs are good enough to detect and measure the residual Helium distribution around the ring. This might be very important, because the vacuum pumps may not detect small Helium leaks in the LHC.

\footnotetext{
${ }^{2}$ In this particular case, the position of the Bremsstrahlungs-target is of interest, and not the quantity of the electron losses.
} 


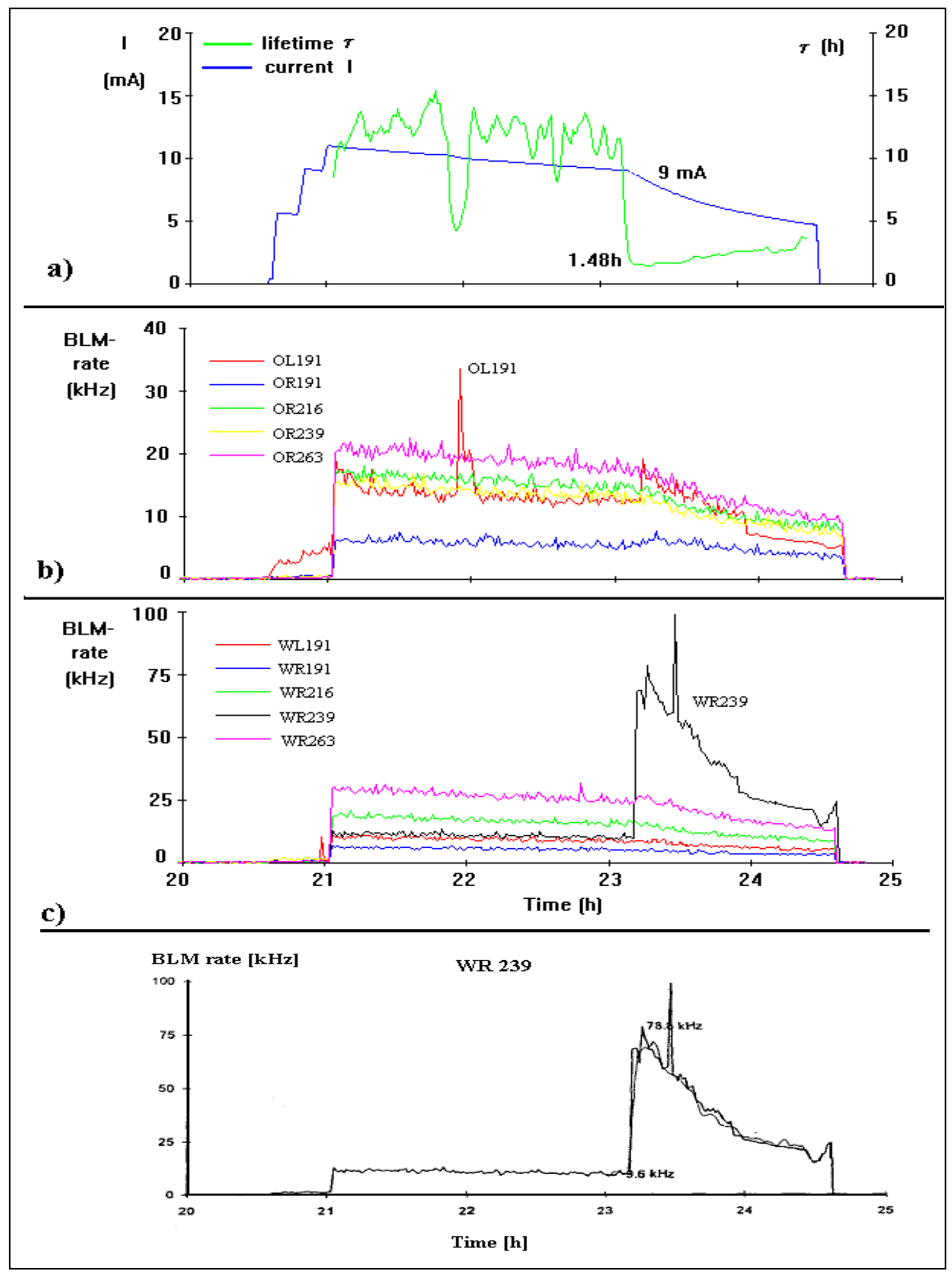

FIGURE 9. Lifetime disruptions in HERAe. a) shows the beam current and the lifetime versus time. The decrease and recovery of the lifetime were different events at different locations in the ring. The positions of the targets in the ring can be defined by the BLMs (b, c). The inverse lifetime and the loss rate of the affected monitor were in very good agreement $(\mathrm{d})$. 
2) Collimators: There are several applications of BLMs at beam collimators. In HERAp they are used to define the setting needed to shield the experiments for background radiation from protons. Ground motion power supply ripple can be detected in the power spectrum of the count rates (Ref. 21, 22). Precise studies of the beam tail were performed at LEP and HERAe, for a better understanding of the beam dynamics (Ref. 23, 24). Even small changes of the beam size can be detected by adjacent BLMs (Ref. 25).

3) Light sources: The PIN diode BLMs are also used in second and third generation light sources. The low energy of the synchrotron radiation together with the good background suppression provides very clear loss detection (Refs. 26, 27).

4) Others: The fast signal of the BLMs make it possible to gate individual bunches to measure the loss distribution of each bunch at the Tevatron (Ref 28). The small size of the BLMs also makes them useable inside High Energy Physics experiments. They are used to measure the background conditions as well as dose measurements. (Ref 29, 30)

\section{ACKNOWLEDGMENTS}

I would like to thank all DESY colleagues, who were involved in the project of PIN diode BLMs at HERA. K. H. Mess encouraged me strongly in the idea of using PIN diodes as a loss detector. The progress in the project was driven by fruitful discussions with him. M. Swars developed the excellent amplifier circuit, which is fast, sensitive and radiation hard enough for the requirements at HERA and elsewhere. He and R. Pforte developed the very reliable counting modules for HERAp and HERAe, respectively. The installation of the proton loss monitors in the HERA tunnel was organized by K. Willmer and S. Schlögl. W. Bialowons instigated the installation of the BLM system in HERAe. F. Ridoutt and the Vacuum group MVP performed its installation. Two diploma theses about the System in HERAp and HERAe were written by S. Schlögl and F. Ridoutt, respectively. Many of the system parameters were discovered in their work. P. Duval, H.G. Wu and M. Lomperski wrote the very useful server, archive and application software, which is intensively used by the operators in the control room.

Special thanks are due to H. Schultz. He maintained the monitors during all the years. The technical care of the system was and is in his very best hands.

Last but not least I would like to thank the BIW2000 organizing committee for the selection of this work for the Faraday Cup Award 2000. 


\section{REFERENCES}

1. J. Bailey, K. H. Mess, K. Wittenburg, private discussions.

2. K. Wittenburg, "Beam Loss Monitors for HERA", PKTR Seminar talk, 1989

3. K. Wittenburg, "Strahlverlustdetektoren für den HERA Protonen - Ring", DESY HERA 1989 - 23 (Nov. 1989), 23 p.

4. K. Wittenburg; "Stralhlenschäden am Photodioden Verstärker"; internal report, PKTR note No. 9 (1987), 7 p.

5. K. Wittenburg, "Radiation Damage in PIN Photodiodes", Nuclear Instruments \& Methodes A270 (1988), p. 56-61 and DESY 87 -070 (July 1987), 18 p.

6. K. Wehrheim, K. Wittenburg, "Radiation Resistance of Beam Loss Monitors", DESY M-94-08 (1994)

7. R. Bacher, J. Bengtsson, G. Bialek, P. Duval, K.H. Mess, V. Nedic, M. Staack, L. Steffen, H.G. Wu (DESY). "The HERA Quench Protection System: A Status Report".DESY-M-96-13A, Jul 1996. In *Sitges 1996, EPAC 96* 2264-2266.

8. F.Ridoutt, "Das Ansprechvermögen des PIN Dioden Strahlverlustmonitors", Internal Note: PKTRnote No. 91 (1993)

9. Eckhart Morré. „Eine Untersuchung für das Zeus-Experiment“.Diploma Thesis, Univ. Hamburg (1992), August 1992, 70 p.

10. H. Fesefeld, "The Simulation of Hadronic Showers - Physics and Application", PITHA 85/02, Aachen, 1985

11. S. Schlögl, "Einsatz von PIN-Photodioden als Protonen-Strahlverlustmonitore bei HERA", Diploma thesis, DESY-HERA-92-03, 79 p.

12. K. Wittenburg, "Preservation of beam loss induced quenches, beam lifetime and beam loss measurements with the HERAp beam loss monitor system“", Nuclear Instruments \& Methods A345 (1994) p. 226 - 229 and DESY 94-003

13. D.R.C. Kelly, Dust in Accelerator Vacuum sysyems. DESY-M-97-10C, Aug 1997. 5pp. and 17th IEEE Particle Accelerator Conference (PAC 97): Accelerator Science, Technology and Applications, Vancouver, Canada, 12-16, May 1997.

14. D.R.C.Kelly, W.Bialowons, K.Wittenburg, DESY HERA Electron-Beam Lifetime Disruption Machine Studies and Observations, Proc. EPAC 1996, Stiges

15. F. Ridoutt, "PIN-Strahlverlustmonitore und ihre Anwendung in dem HERA-Elektronen-Ring", DESY-HERA 95-08, Diploma thesis

16. W. Bialowons, K. Wittenburg, F. Ridoutt, "Electron Beam Loss Monitors for HERA", Proc. 4. EPAC 1994, LONDON

17. F. Ridoutt, K. Wittenburg, "Experience with the Electron and Proton Beam Loss monitor System at HERA", Proc. EPAC 1996, Stiges

18. K. Wittenburg, "Reduction of the Sensitivity of PIN Diode Beam Loss Monitors to Synchrotron Radiation by Use of a Copper Inlay", DESY HERA 96-06

19. K. Wittenburg, "Improvements of the HERA Electron Beam Loss Monitor System", in "HERA Accelerator Studies 1999", DESY HERA 00-02, G. Hoffstaetter (ed.)

20. T. Spickermann, K. Wittenburg, "Simulation of Point Beam Losses in LHC Superconducting Magnets", LHC Project Note 124, 1997, 15 p.

21. O.S. Bruning, K.H. Mess, M. Seidel, F. Willeke, "Measuring the Effect of an External Tune Modulation on the Particle Diffusion in the Proton Storage Ring HERA", DESY-HERA-94-01, Jan 1994. 22pp.

22. K.H. Mess, M. Seidel, "Measurement of Proton Beam Oszillations at low Frequencies", London 1994, Proceedings, EPAC 94, vol. 2, p. 1731-1733.

23. H. Burkhardt, I. Reichel, G. Roy, "Transverse Beam Tails due to Inelastic Scattering", CERN-SL99-068 (OP)

24. Atoosa Meseck, "Tail Scan Experiments I + II at the HERA Electron Ring", DESY HERA 00-02, G. Hoffstaetter (ed.)

25. T. Spickermann, K. Wittenburg, "Improvements in the useful dynamic Range of the LEP Beam Loss Monitors", CERN SL-note 97-05, 1997, 16 p.

26. K.T. Hsu et al. "Real-Time Beam Loss Monitoring and its Applications in SRRC" Proceedings of the 1997 Particle Accelerator ConferenceVancouver, B.C., Canada, 12-16 May 1997

27. J. Hinkson, “ALS Beam Instrumentation, Beam Loss Monitoring”, Feb. 1999

28. Vladimir Shiltsev, "Fast PIN-Diode Beam Loss Monitors at Tevatron", FERMILAB-TM-2012, Jul 1997. 22 p.

29. E. Moree, "Ein Untergrundmonitor für das Zeus Experiment", 1992. Diploma thesis Universtät Hamburg, and J. Ewert, H. Spitzer, G.G. Winter, "Background Measurements at HERA near the H1 Detector". 1993, DESY - Internal Rep. FH1T-93-02 (93/04,rec.May) 19 p.

30. A Drees, BNL-RHIC, privat communication 